\title{
Comparison of Level of Fatigue and Disease Correlates in Patients With Rheumatoid Arthritis and Systemic Sclerosis
}

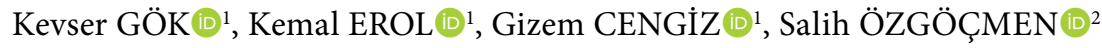 \\ ${ }^{1}$ Department of Physical Medicine and Rehabilitation, Division of Rheumatology, Erciyes University Faculty of Medicine, Kayseri, Turkey \\ ${ }^{2}$ Department of Rheumatology, Medical Park Gaziosmanpaşa Hospital, İstanbul, Turkey
}

\begin{abstract}
Objectives: This study aims to compare the levels of fatigue in patients with rheumatoid arthritis (RA) and systemic sclerosis (SSc) and to assess the potential influence of fatigue on clinical variables.

Patients and methods: Age- and sex-matched adult patients with SSc ( $n=50 ; 6$ males, 44 females; mean age 47.7 years; range 23 to 72 years) and RA ( $n=51 ; 6$ males, 45 females; mean age 50.8 years; range 23 to 71 years) were consecutively recruited. Patients were examined and evaluated for disease specific and generic outcome measures including disease activity parameters, physical functions, psychological status, and health related quality of life (HRQoL) measures. Level of fatigue was assessed by Fatigue Severity Scale and Multidimensional Assessment of Fatigue scale. These were interviewed by the same experienced physician who was blind to clinical data.

Results: Patients had similar educational and smoking status, as well as functioning and HRQoL. However, patients with RA declared higher levels on Visual Analog Scale-pain $(p=0.012)$ and higher Body Mass Index than patients with SSc $(p<0.0001)$ and lower distances in six-minute walking test $(p=0.002)$. Levels of fatigue were quite similar between patients with RA and SSc. Levels of fatigue, measured by different scales, were significantly correlated with physical functions and HRQoL measures and psychometric variables in both groups; however, the correlation coefficients were relatively higher in patients with RA.

Conclusion: Fatigue is a major problem in patients with RA and SSc. Our findings revealed that level of fatigue was quite similar between patients with RA and SSC and significantly correlated with physical functions and HRQoL. Patients with RA and SSC should be carefully evaluated for fatigue by using valid scales and the impact of fatigue on clinical measures should not be overlooked.

Keywords: Fatigue; quality of life; rheumatoid arthritis; systemic sclerosis.
\end{abstract}

Rheumatoid arthritis (RA) is an autoimmune, systemic, and chronic inflammatory disease causing pain, joint destruction, deformities, and disability. ${ }^{1}$

Systemic sclerosis (SSc) is a chronic autoimmune disease characterized by inflammation, vasculopathy, and fibrosis of the skin and internal organs. ${ }^{2}$ SSc may cause damage in multiple organ systems, including the gastrointestinal tract, respiratory, and cardiovascular systems. RA and SSc are two different forms of inflammatory diseases associated with significant morbidity and increased mortality and both diseases are more common in females. ${ }^{3-5}$

Fatigue is a common and disabling symptom in autoimmune rheumatic disorders including RA and SSc; however, assessment of fatigue is often overlooked.., 7 The pathophysiology of fatigue in $\mathrm{RA}$ and SSc is not well understood, but is thought to be multifactorial (including increased pain, poor physical function, sleep quality, and psychosocial factors). ${ }^{8-12}$

Received: October 13, 2017 Accepted: November 14, 2017 Published online: January 15, 2018

Correspondence: Kevser Gök, MD. Erciyes Üniversitesi Tıp Fakültesi Fiziksel Tıp ve Rehabilitasyon Anabilim Dalı, Romatoloji Bilim Dalı, 38039 Melikgazi, Kayseri, Turkey. Tel: +90 352 - 2076666 e-mail: kevserorhangok@gmail.com

\section{Citation:}

Gök K, Erol K, Cengiz G, Özgöçmen S. Comparison of Level of Fatigue and Disease Correlates in Patients With Rheumatoid Arthritis and Systemic Sclerosis. Arch Rheumatol 2018;33(3):316-321. 
Although fatigue is not a mandatory manifestation for the diagnosis of RA or SSc, persistent fatigue is an important clinical problem which may interfere with loss of functioning and health related quality of life (HRQoL) and contributes to the increased disease burden. ${ }^{13-15}$ Several studies compared these two diseases in terms of clinical, laboratory and imaging aspects; however, level of fatigue which was assessed by single-item and multidimensional scales have not been evaluated comparatively. Therefore, in this study, we aimed to compare the levels of fatigue in RA and SSc and to assess the potential influence of fatigue on clinical variables.

\section{PATIENTS AND METHODS}

Age- and sex-matched patients with SSc $(n=50$; 6 males, 44 females; mean age 47.7 years; range 23 to 72 years) and $\mathrm{RA}(\mathrm{n}=51 ; 6$ males, 45 females; mean age 50.8 years; range 23 to 71 years) were consecutively recruited at Erciyes University Faculty of Medicine between September 2015 and January 2016. Patients met the American College of Rheumatology/European League Against Rheumatism criteria for SSc and RA. ${ }^{16,17}$

Patients with a prior diagnosis of psychiatric disorders or fibromyalgia or taking medications for these disorders, or who had uncontrolled diabetes or neurological disorders were excluded. All patients were biologic naive. Patients were examined and evaluated for disease specific and generic outcome measures including disease activity parameters, physical functions, psychological status, and HRQoL measures. Six-minute walking distance was noted in meters.
Visual Analog Scale of pain (VAS-pain, 0-10 scale) within the last week was noted. Level of fatigue was assessed by Fatigue Severity Scale (FSS) and Multidimensional Assessment of Fatigue (MAF) scale, Short-Form 36 (SF-36)-vitality and VAS of fatigue (0-10 numeric scale). These were interviewed by the same experienced physician who was blind to clinical data.

The FSS and MAF are self-administered questionnaires to measure self-reported fatigue. FSS questionnaire contains nine statements that attempt to detect severity of fatigue and its effects on motivation, exercise, physical functioning, carrying out duties, interfering with work, family, or social life. This scale is a seven-point Likert scale: 1 (strongly disagree) and 7 (strongly agree). The scoring is performed by calculating the average of responses ranging from 1 to 9 . Higher scores indicate more severe fatigue. ${ }^{18}$ The questionnaire takes nearly two-three minutes to complete. Validity and reliability of Turkish version of FSS to assess fatigue have been shown in patients with fibromyalgia and multiple sclerosis. ${ }^{19,20}$

The MAF questionnaire is used to measure four dimensions of fatigue in the past week; severity, distress, timing, and degree of interference with activities of daily living (household chores, cooking, bathing, dressing, working, socializing, sexual activity, leisure and recreational activities, shopping, walking, and other exercising). The MAF consists of 16 items (severity [items 1 and 2], distress [item 3], degree of interference in activities of daily living [items 4-14], and timing [items 15 and 16]). Only the first 15 items are used to calculate the Global Fatigue Index. ${ }^{21}$ The Turkish version of MAF is valid and reliable to detect the severity of

Table 1. Patients' demographic and clinical data

\begin{tabular}{|c|c|c|c|}
\hline & Systemic sclerosis $(\mathrm{n}=50)$ & Rheumatoid arthritis $(\mathrm{n}=51)$ & \\
\hline & Mean \pm SD & Mean \pm SD & $p$ \\
\hline Age (year) & $47.7 \pm 13.1$ & $50.8 \pm 10.1$ & 0.189 \\
\hline Body Mass Index $\left(\mathrm{kg} / \mathrm{m}^{2}\right)$ & $26.3 \pm 6.0$ & $31.0 \pm 6.2$ & $<0.0001$ \\
\hline Symptom duration (years) & $10.1 \pm 7.0$ & $11.1 \pm 8.0$ & 0.525 \\
\hline Visual Analog Scale-pain & $4.0 \pm 2.9$ & $5.3 \pm 2.1$ & 0.012 \\
\hline Six-minute walking distance (m) & $423.4 \pm 76.8$ & $372.9 \pm 83.4$ & 0.002 \\
\hline Health Assessment Questionnaire & $0.5 \pm 0.6$ & $0.5 \pm 0.6$ & 0.946 \\
\hline Short-form 36 physical component score & $50.8 \pm 20.9$ & $50.7 \pm 21.3$ & 0.983 \\
\hline Short-form 36 mental component score & $58.3 \pm 21.5$ & $56.7 \pm 22.6$ & 0.719 \\
\hline
\end{tabular}


Table 2. Patients' fatigue data

\begin{tabular}{|c|c|c|c|}
\hline & Systemic sclerosis $(n=50)$ & Rheumatoid arthritis $(n=51)$ & \\
\hline & Mean \pm SD & Mean \pm SD & $p$ \\
\hline Fatigue severity scale & $4.7 \pm 1.8$ & $4.4 \pm 1.9$ & 0.439 \\
\hline Multidimensional Assessment of Fatigue-severity & $6.4 \pm 2.1$ & $5.5 \pm 2.4$ & 0.064 \\
\hline Multidimensional Assessment of Fatigue-distress & $5.8 \pm 2.7$ & $5.6 \pm 2.6$ & 0.656 \\
\hline Multidimensional Assessment of Fatigue-interference ADL & $4.8 \pm 2.3$ & $5.1 \pm 2.2$ & 0.534 \\
\hline Multidimensional Assessment of Fatigue-timing & $6.4 \pm 2.1$ & $6.6 \pm 2.1$ & 0.696 \\
\hline Multidimensional Assessment of Fatigue-GFI & $27.8 \pm 12.2$ & $25.3 \pm 12.8$ & 0.317 \\
\hline Visual Analog Scale-fatigue & $6.0 \pm 2.7$ & $5.1 \pm 2.8$ & 0.148 \\
\hline Short form 36-vitality & $48.4 \pm 20.3$ & $51.8 \pm 21.5$ & 0.421 \\
\hline
\end{tabular}

fatigue in patients with chronic musculoskeletal disorders. ${ }^{22}$ FSS and MAF subscales have high reproducibility and correlated well with QoL in patients with SSc and Turkish versions are appropriate to assess fatigue in patients with SSc. ${ }^{23}$ The study protocol was approved by the Erciyes University Faculty of Medicine Ethics Committee. A written informed consent was obtained from each patient. The study was conducted in accordance with the principles of the Declaration of Helsinki.

\section{Statistical analysis}

All analyses were performed on a personal computer using IBM SPSS version 22.0 (IBM Corp., Armonk, NY, USA). Normality of distributions was assessed by KolmogorovSmirnov test. Parameters and demographic variables in patients with $\mathrm{RA}$ and $\mathrm{SSc}$ were compared using t-test or chi-square test. Relationship between parameters was analyzed by Spearman or Pearson correlation tests. A $p$ value of $<0.05$ was considered statistically significant.

\section{RESULTS}

Patients had similar age and sex, symptom duration, educational and smoking status, as well as functioning (Health Assessment Questionnaire) and health related quality of life (SF-36) (Table 1). Comorbid diseases (including controlled diabetes, hypertension, atherosclerotic heart disease, thyroid diseases alone or in combination) were present in $60.8 \%$ of patients with RA and $58.0 \%$ of patients

Table 3. Correlation matrix between Multidimensional Assessment of Fatigue, Fatigue Severity Scale, Visual Analog Scale-fatigue, Health Assessment Questionnaire, and Short-Form 36

\begin{tabular}{|c|c|c|c|c|c|}
\hline & VAS-fatigue & HAQ & SF-36-Vitality & SF-36-PCS & SF-36-MCS \\
\hline \multicolumn{6}{|l|}{ Systemic sclerosis } \\
\hline Fatigue severity scale & $0.59^{* * *}$ & $0.39 * *$ & -0.22 & $-0.38^{* *}$ & $-0.32^{*}$ \\
\hline Multidimensional Assessment of Fatigue-severity & $0.98^{* * *}$ & $0.45^{* *}$ & -0.28 & $-0.49^{* *}$ & -0.26 \\
\hline Multidimensional Assessment of Fatigue-distress & $0.78^{* *}$ & $0.49^{* *}$ & -0.20 & $-0.44^{* * *}$ & $-0.29^{*}$ \\
\hline Multidimensional Assessment of Fatigue-interference ADL & $0.61^{* *}$ & $0.39^{* *}$ & -0.24 & $-0.41^{* *}$ & $-0.35^{*}$ \\
\hline Multidimensional Assessment of Fatigue-timing & $0.49^{* * *}$ & 0.28 & $-0.34^{*}$ & $-0.40^{* *}$ & $-0.39^{* *}$ \\
\hline Multidimensional Assessment of Fatigue-GFI & $0.95^{* *}$ & $0.48^{* *}$ & $-0.39^{* *}$ & $-0.65^{* *}$ & $-0.51^{* *}$ \\
\hline Visual Analog Scale-fatigue & 1 & $0.44^{* *}$ & $-0.42^{* * *}$ & $-0.68^{* *}$ & $-0.52^{* *}$ \\
\hline \multicolumn{6}{|l|}{ Rheumatoid arthritis } \\
\hline Fatigue severity scale & $0.84^{* *}$ & 0.24 & $-0.44^{* * *}$ & $-0.62^{* * *}$ & $-0.64^{* * *}$ \\
\hline Multidimensional Assessment of Fatigue-severity & $0.97^{* * *}$ & $0.44^{* * *}$ & $-0.53^{* *}$ & $-0.47^{* *}$ & $-0.59^{* *}$ \\
\hline Multidimensional Assessment of Fatigue-distress & $0.92^{* * *}$ & $0.47^{* *}$ & $-0.49^{* * *}$ & $-0.50^{* * *}$ & $-0.58^{* *}$ \\
\hline Multidimensional Assessment of Fatigue-interference ADL & $0.58^{* * *}$ & 0.14 & $-0.36^{*}$ & $-0.32 *$ & $-0.43^{* * *}$ \\
\hline Multidimensional Assessment of Fatigue-timing & $0.32^{*}$ & -0.15 & $-0.32^{*}$ & $-0.33^{*}$ & $-0.42^{* *}$ \\
\hline Multidimensional Assessment of Fatigue-GFI & $0.94^{* *}$ & $0.41^{* *}$ & $-0.50^{* * *}$ & $-0.55^{* * *}$ & $-0.62^{* * *}$ \\
\hline Visual Analog Scale-fatigue & 1 & $0.45^{* *}$ & $-0.46^{* *}$ & $-0.52^{* * *}$ & $-0.57^{* * *}$ \\
\hline
\end{tabular}

VAS: Visual Analog Scale; HAQ: Health Assessment Questionnaire; SF-36: Short-Form 36; SF-36-PCS: Short-Form 36 physical component score; SF-36-MCS: Short-Form 36 mental component score; ADL: Activities of daily living; GFI: Global fatigue index; ${ }^{*} \mathrm{p}<0.05 ;{ }^{* *} \mathrm{p}<0.01$ 
with SSc ( $p>0.05)$. Mean hemoglobin level was $13.2 \pm 1.7$ in patients with RA and $12.8 \pm 1.5$ in patients with $\mathrm{SSc}$ and was quite similar between groups $(p=0.182)$. Anti-citrullinated protein antibodies were positive in $52.9 \%$ of patients with RA, and $6.0 \%$ of patients with SSc. Mean disease activity score 28 was $4.0 \pm 1.1$ in patients with RA. Mean modified Rodnan skin score was $13.0 \pm 8.1$ in patients with SSc. Twentytwo patients (44\%) had diffuse and 28 patients (56\%) had limited type SSc.

However, patients with RA had higher levels on VAS-pain $(5.3 \pm 2.1$ vs. $4.0 \pm 2.9, \mathrm{p}=0.012)$, and higher body mass index $(31.0 \pm 6.2$ vs. $26.3 \pm 6.0, p<0.0001)$ and lower distances in sixminute walking test $(372.9 \pm 83.4$ vs. $423.4 \pm 76.8$, $\mathrm{p}=0.002$ ) than patients with SSc (Table 1).

Levels of fatigue which were assessed by VAS, SF-36-vitality, FSS, and MAF were quite similar between patients with RA and SSc (Table 2). Levels of fatigue, measured by different scales, were significantly correlated with physical functions and HRQoL measures and psychometric variables in both groups; however, the correlation coefficients were relatively higher in patients with RA (Table 3).

\section{DISCUSSION}

In our study, we compared the level of fatigue in $\mathrm{RA}$ and SSc and assessed its potential influence on clinical variables. We showed that the level of fatigue was quite similar between patients with RA and SSc and significantly correlated with physical functions and HRQoL.

Fatigue is a major problem in patients with $\mathrm{RA}$ and SSc, and has a negative impact on HRQoL. ${ }^{13,15}$ Although fatigue is a common and disturbing symptom in patients with RA and $\mathrm{SSc}$, assessment of fatigue is often ignored by physicians. ${ }^{6,7}$ Various questionnaires including single-item, multi-item, and multidimensional questionnaires were used to evaluate fatigue in patients with RA and SSc. Up to date, no consensus was reached on which questionnaire has to be used in RA or SSc to assess fatigue. Although Wolfe suggested that single-item VAS was similar or better than longer questionnaires in $\mathrm{RA},{ }^{24}$ the common practice is in favor of using specific, multi-item and multidimensional scales of fatigue. ${ }^{25,26}$ In our study, the level of fatigue was assessed by using Turkish versions of MAF, FSS, SF-36-vitality subscore, and VAS of fatigue. To our knowledge, this is the first report comparing these two diseases in terms of fatigue by using various assessment tools.

The mechanism that leads to fatigue in RA and SSc is multifactorial. Pharmacological and non-pharmacological treatments were associated with decreased fatigue levels. ${ }^{27-29}$ Small number of studies reported the effect of biologic therapies on fatigue levels in patients with RA. In a recent meta-analysis, Chauffier et al. ${ }^{30}$ reported the effect of biologic agents on fatigue in RA; however, underscored that this effect was rather minor. Additionally, Pollard et al. ${ }^{28}$ showed reduced fatigue levels with disease modifying antirheumatic drug treatment.

Furthermore, Mancuso et al. ${ }^{31}$ suggested that fatigue severity (FSS scores) was associated with increased disability, anxiety and depression symptom, less social support and more social stress in patients with RA. Another study revealed that fatigue was strongly associated with functional disability, increased pain and depression symptoms, poor sleep, and lower hematocrit levels. ${ }^{8}$ In patients with RA, increased pain, depression symptoms, female sex, longer symptom duration, ${ }^{9}$ and sleep disturbances were shown as the significant predictors of increased fatigue. ${ }^{11,21}$ Nicassio et al. ${ }^{32}$ showed that higher levels of disease activity, mood disturbance, and poor sleep quality had direct effect on fatigue, and also discussed the indirect cause and effect relationships of these factors on fatigue.

In SSc, cutaneous and internal organ involvements are the primary targets of treatment and unfortunately fatigue is generally an overlooked feature to assess or manage by clinicians. A few studies revealed the association of fatigue with decreased physical functions, sleep quality, increased pain, and depression symptoms in patients with SSc. ${ }^{12,33}$ Canadian Scleroderma Research Group reported that higher fatigue was significantly associated with the number of medical comorbidities, breathing problems, gastrointestinal symptoms, and current smoking. ${ }^{34}$ Thombs et al. ${ }^{7}$ showed that fatigue level in patients with SSc was higher 
than general population and cancer patients in remission; however, similar to patients under active treatment for cancer and patients with other rheumatic diseases.

Our study had some limitations. First, the number of patients was relatively small. We excluded patients with a history of or under treatment with biologics for the fact that these agents had major impact on fatigue levels. Secondly, we could only assess patients in a cross-sectional design. More robust results on fatigue and its correlation with clinical findings may be warranted in a longitudinal design. Although our study populations were matched for sex, number of male patients was relatively small which prohibited assessing sex specific differences on fatigue in separate sex groups.

In conclusion, patients with RA and SSc should be carefully evaluated for fatigue by using valid scales and the impact of fatigue on clinical measures should not be overlooked. Longitudinal follow-up of patients with different treatments may enhance our understanding for the possible cause and effect relationship of fatigue with other clinical manifestations.

\section{Declaration of conflicting interests}

The authors declared no conflicts of interest with respect to the authorship and/or publication of this article.

\section{Funding}

The authors received no financial support for the research and/or authorship of this article.

\section{REFERENCES}

1. Grassi W, De Angelis R, Lamanna G, Cervini C. The clinical features of rheumatoid arthritis. Eur J Radiol 1998;27:18-24.

2. Denton CP. Systemic sclerosis: from pathogenesis to targeted therapy. Clin Exp Rheumatol 2015;33:3-7.

3. Medsger TA Jr. Epidemiology of systemic sclerosis. Clin Dermatol 1994;12:207-16.

4. Ioannidis JP, Vlachoyiannopoulos PG, Haidich AB, Medsger TA Jr, Lucas M, Michet CJ, et al. Mortality in systemic sclerosis: an international meta-analysis of individual patient data. Am J Med 2005;118:2-10.

5. Alamanos Y, Drosos AA. Epidemiology of adult rheumatoid arthritis. Autoimmun Rev 2005;4:130-6.

6. Tack BB. Fatigue in rheumatoid arthritis. Conditions, strategies, and consequences. Arthritis Care Res
1990;3:65-70.

7. Thombs BD, Bassel M, McGuire L, Smith MT, Hudson M, Haythornthwaite JA. A systematic comparison of fatigue levels in systemic sclerosis with general population, cancer and rheumatic disease samples. Rheumatology (Oxford) 2008;47:1559-63.

8. Belza BL. Comparison of self-reported fatigue in rheumatoid arthritis and controls. J Rheumatol 1995;22:639-43.

9. Huyser BA, Parker JC, Thoreson R, Smarr KL, Johnson JC, Hoffman R. Predictors of subjective fatigue among individuals with rheumatoid arthritis. Arthritis Rheum 1998;41:2230-7.

10. Riemsma RP, Rasker JJ, Taal E, Griep EN, Wouters $\mathrm{JM}$, Wiegman $\mathrm{O}$. Fatigue in rheumatoid arthritis: the role of self-efficacy and problematic social support. $\mathrm{Br}$ J Rheumatol 1998;37:1042-6.

11. van Hoogmoed D, Fransen J, Bleijenberg G, van Riel P. Physical and psychosocial correlates of severe fatigue in rheumatoid arthritis. Rheumatology (Oxford) 2010;49:1294-302.

12. Sandusky SB, McGuire L, Smith MT, Wigley FM, Haythornthwaite JA. Fatigue: an overlooked determinant of physical function in scleroderma. Rheumatology (Oxford) 2009;48:165-9.

13. Rupp I, Boshuizen HC, Jacobi CE, Dinant HJ, van den Bos GA. Impact of fatigue on health-related quality of life in rheumatoid arthritis. Arthritis Rheum 2004;51:578-85.

14. Suarez-Almazor ME, Kallen MA, Roundtree AK, Mayes M. Disease and symptom burden in systemic sclerosis: a patient perspective. $J$ Rheumatol 2007;34:1718-26.

15. Ibn Yacoub Y, Amine B, Bensabbah R, Hajjaj-Hassouni $\mathrm{N}$. Assessment of fatigue and its relationships with disease-related parameters in patients with systemic sclerosis. Clin Rheumatol 2012;31:655-60.

16. van den Hoogen F, Khanna D, Fransen J, Johnson SR, Baron M, Tyndall A, et al. 2013 classification criteria for systemic sclerosis: an American College of Rheumatology/European League against Rheumatism collaborative initiative. Arthritis Rheum 2013;65:2737-47.

17. Aletaha D, Neogi T, Silman AJ, Funovits J, Felson DT, Bingham CO, et al. 2010 rheumatoid arthritis classification criteria: an American College of Rheumatology/European League Against Rheumatism collaborative initiative. Ann Rheum Dis 2010;69:1580-8.

18. Krupp LB, LaRocca NG, Muir-Nash J, Steinberg AD. The fatigue severity scale. Application to patients with multiple sclerosis and systemic lupus erythematosus. Arch Neurol 1989;46:1121-3.

19. Armutlu K, Korkmaz NC, Keser I, Sumbuloglu V, Akbiyik DI, Guney Z, et al. The validity and reliability of the Fatigue Severity Scale in Turkish multiple sclerosis patients. Int $\mathrm{J}$ Rehabil Res 2007;30:81-5. 
20. Gencay-Can A, Can SS. Validation of the Turkish version of the fatigue severity scale in patients with fibromyalgia. Rheumatol Int 2012;32:27-31.

21. Belza BL, Henke CJ, Yelin EH, Epstein WV, Gilliss CL. Correlates of fatigue in older adults with rheumatoid arthritis. Nurs Res 1993;42:93-9.

22. Yildirim Y, Ergin G. A validity and reliability study of the Turkish Multidimensional Assessment of Fatigue (MAF) scale in chronic musculoskeletal physical therapy patients. J Back Musculoskelet Rehabil 2013;26:307-16.

23. Gok K, Cengiz G, Erol K, Ozgocmen S. The Turkish version of multidimensional assessment of fatigue and fatigue severity scale is reproducible and correlated with other outcome measures in patients with systemic sclerosis. Arch Rheumatol 2016;31:329-32.

24. Wolfe F. Fatigue assessments in rheumatoid arthritis: comparative performance of visual analog scales and longer fatigue questionnaires in 7760 patients. J Rheumatol 2004;31:1896-902.

25. Nikolaus S, Bode C, Taal E, van de Laar MA. Which dimensions of fatigue should be measured in patients with rheumatoid arthritis? A Delphi study. Musculoskeletal Care 2012;10:13-7.

26. Oude Voshaar MA, Ten Klooster PM, Bode C, Vonkeman HE, Glas CA, Jansen $\mathrm{T}$, et al. Assessment of fatigue in rheumatoid arthritis: a psychometric comparison of single-item, multiitem, and multidimensional measures. $\mathrm{J}$ Rheumatol 2015;42:413-20.

27. Strand V, Singh JA. Improved health-related quality of life with effective disease-modifying antirheumatic drugs: evidence from randomized controlled trials.
Am J Manag Care 2007;13 Suppl 9:S237-51.

28. Pollard LC, Choy EH, Gonzalez J, Khoshaba B, Scott DL. Fatigue in rheumatoid arthritis reflects pain, not disease activity. Rheumatology (Oxford) 2006;45:885-9.

29. Neill J, Belan I, Ried K. Effectiveness of nonpharmacological interventions for fatigue in adults with multiple sclerosis, rheumatoid arthritis, or systemic lupus erythematosus: a systematic review. J Adv Nurs 2006;56:617-35.

30. Chauffier K, Salliot C, Berenbaum F, Sellam J. Effect of biotherapies on fatigue in rheumatoid arthritis: a systematic review of the literature and meta-analysis. Rheumatology (Oxford) 2012;51:60-8.

31. Mancuso CA, Rincon M, Sayles W, Paget SA. Psychosocial variables and fatigue: a longitudinal study comparing individuals with rheumatoid arthritis and healthy controls. J Rheumatol 2006;33:1496-502.

32. Nicassio PM, Ormseth SR, Custodio MK, Irwin MR, Olmstead R, Weisman MH. A multidimensional model of fatigue in patients with rheumatoid arthritis. $\mathrm{J}$ Rheumatol 2012;39:1807-13.

33. Nikolaus S, Bode C, Taal E, van de Laar MA. Fatigue and factors related to fatigue in rheumatoid arthritis: a systematic review. Arthritis Care Res (Hoboken) 2013;65:1128-46.

34. Thombs BD, Hudson M, Bassel M, Taillefer SS, Baron M. Sociodemographic, disease, and symptom correlates of fatigue in systemic sclerosis: evidence from a sample of 659 Canadian Scleroderma Research Group Registry patients. Arthritis Rheum 2009;61:966-73. 